\title{
What can Rapid Terrestrial Biogenesis Tell Us about Life in the Universe?
}

\author{
Charles H. Lineweaver \& Tamara M. Davis \\ School of Physics, University of New South Wales and the \\ Australian Centre for Astrobiolgy, Sydney, Australia \\ charley@bat.phys.unsw.edu.au
}

\begin{abstract}
It is sometimes asserted that the rapidity of biogenesis on Earth suggests that life is common in the Universe. We critically examine the assumptions inherent in this argument. Using a lottery model for biogenesis in the Universe, we convert the observational constraints on the rapidity of biogenesis on Earth into constraints on the probability of biogenesis on other terrestrial planets. For example, if terrestrial biogenesis took less than $200 \mathrm{Myr}$ (and we assume that it could have taken 1 billion years) then we find the probability of biogenesis on terrestrial planets older than $\sim 1 \mathrm{Gyr}$, is $>36 \%$ at the $95 \%$ confidence level. However, there are assumptions and selection effects that complicate this result: although we correct the analysis for the fact that biogenesis is a prerequisite for our existence, our result depends on the plausible assumption that rapid biogenesis is not such a prerequisite.
\end{abstract}

Our existence on Earth can tell us little about how common life is in the Universe or about the probability of biogenesis on a terrestrial planet because, even if this probability were infinitesimally small and there were only one lifeharboring planet in the Universe we would, of necessity, find ourselves on that planet. However, the rapidity with which life appeared on Earth gives us more information. We find ourselves in the group of planets on which biogenesis has necessarily occurred - we have of necessity won the biogenetic lottery some time in the past. And we also find that biogenesis has occurred rapidly - we won soon after the tickets went on sale.

From radioactivity to lotteries, more probable things happen more rapidly. If life were rare it would be unlikely that biogenesis would have occurred as rapidly as it seems to have occurred on Earth.

During and immediately following the Earth's formation there was a period without life ( $\left.\Delta t_{\text {frustrated }}\right)$, followed by a period during which life evolved $\left(\Delta t_{\text {biogenesis }}\right)$, followed by a period during which life has been present $\left(\Delta t_{\text {life }}\right)$. The sum of these intervals adds up to the age of the Earth (Fig. 1):

$$
\Delta t_{\text {frustrated }}+\Delta t_{\text {biogenesis }}+\Delta t_{\text {life }}=\Delta t_{\text {Earth }}
$$

Inserting observational estimates for these durations (Lineweaver \& Davis 2002, hereafter LD) yields

$$
0.5 \pm 0.4+\Delta t_{\text {biogenesis }}+4.0_{-0.2}^{+0.4}=4.566 \pm 0.002 \mathrm{Gyr}
$$




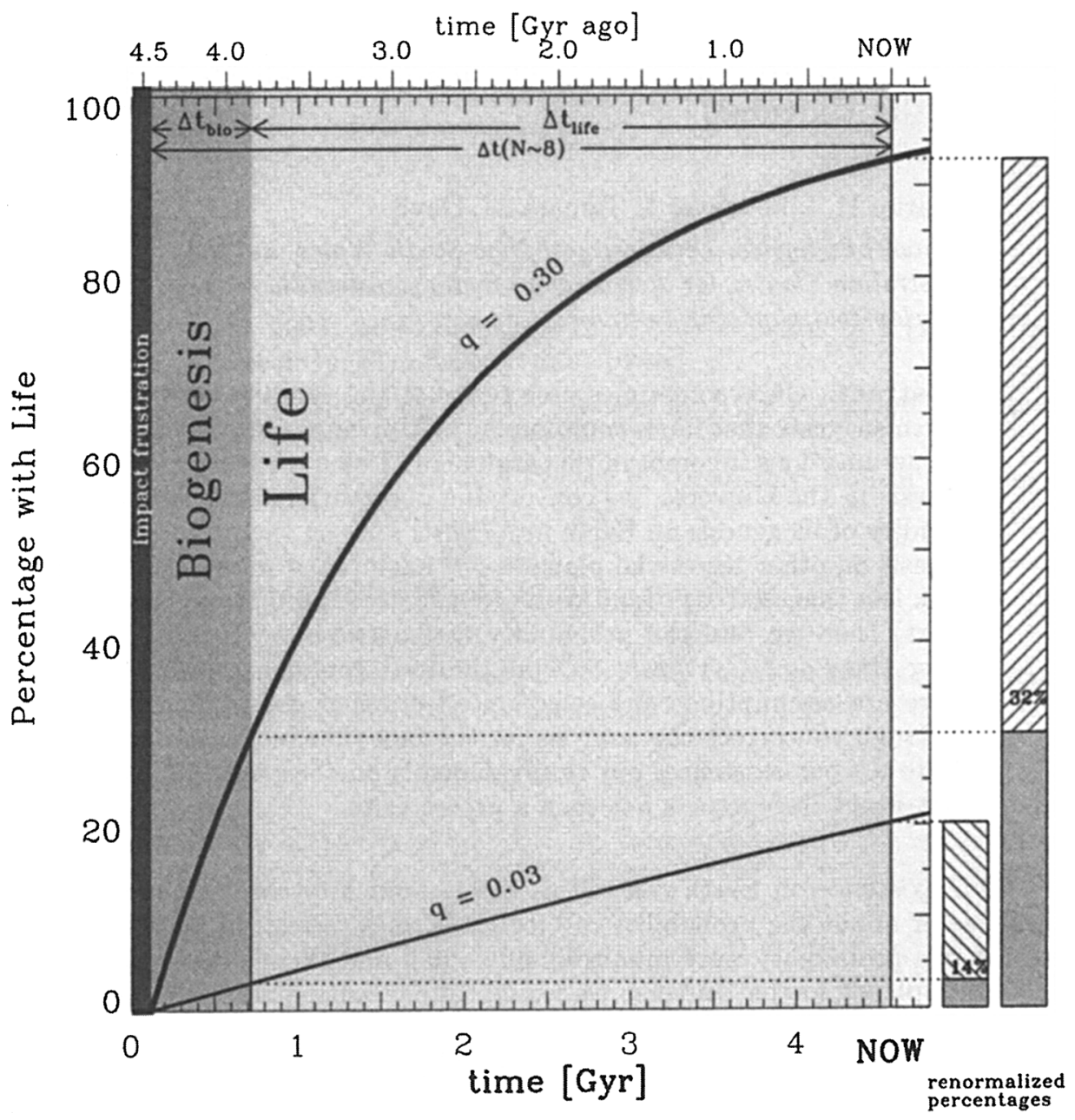

Figure 1. We divide the history of the Earth into three epochs: Impact frustration, Biogenesis and Life. Consider a group of terrestrial planets with approximately the same probability of biogenesis ' $q$ ' as Earth. Suppose $q=0.30$. At their formation, none of these planets had life. As time passed, life arose on more and more of them (thick line) After $\Delta t_{\text {biogenesis }}, 30 \%$ will have life (that is how $q=0.30$ is defined). After $4.566 \mathrm{Gyr}, 93 \%$ will have life (7\% still will not). If $q$ is high $(0.30$, thick line) a large fraction $(32 \%)$ of the planets that have evolved life within $4.566 \mathrm{Gyr}$ of formation, have life that evolved rapidly - within $\Delta t_{\text {biogenesis }}$ - on their planets. If $q$ is low $(0.03$, thin line) then a smaller fraction (14\%) will have life that evolved rapidly. These different percentages illustrate the principle that a single observation of rapid terrestrial biogenesis is more likely to be the result of high $q$ and allows us to compute the relative likelihood of $q$. 
or

$$
\Delta t_{\text {biogenesis }}=0.1_{-0.1}^{+0.5} \mathrm{Gyr} \text {. }
$$

Thus we take $600 \mathrm{Myr}$ as a crude estimate of the upper limit for the time it took life to appear on Earth. This is the basis for the statement that biogenesis occurred rapidly.

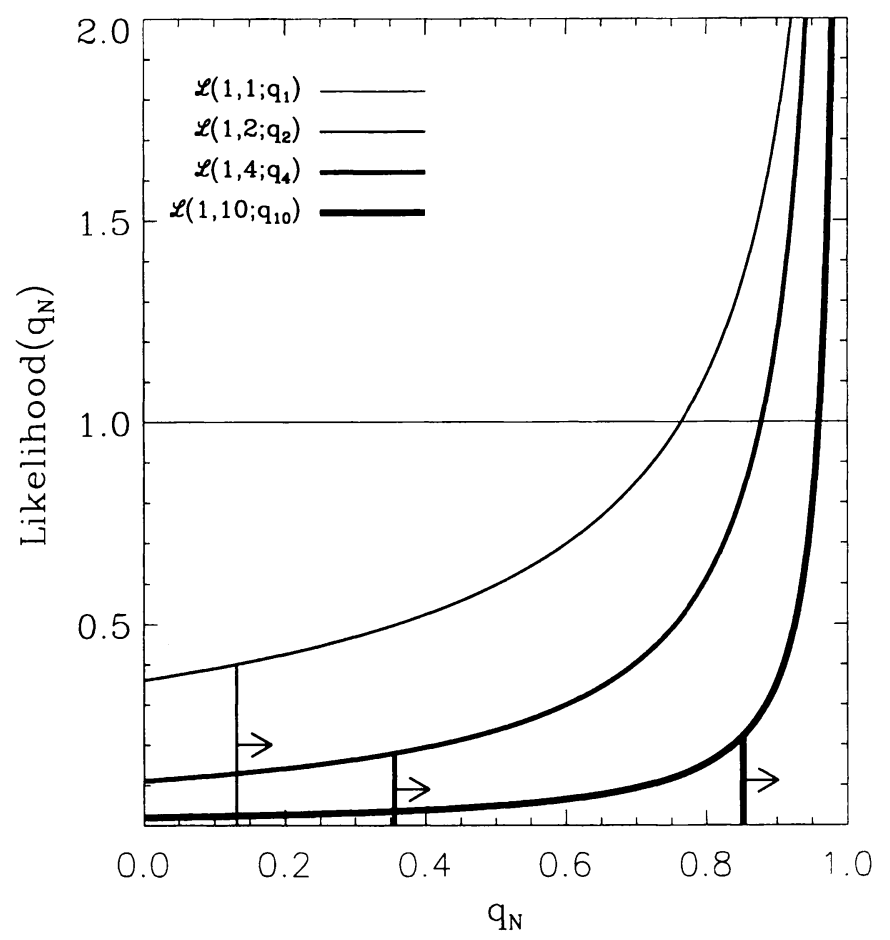

Figure 2. Likelihood of $q_{N}$. Here we generalize inferences about $q$ (the probability of biogenesis within $\Delta t_{\text {biogenesis }}$ ) to inferences about $q_{N}$ (the probability of biogenesis within an arbitrary time, $\Delta t(N)=$ $\left.N \times \Delta t_{\text {biogenesis }}\right)$. The $95 \%$ lower limit on $q_{N}$ increases dramatically as $N$ increases, constraining $q_{N}$ to be close to 1 . Specifically, we only need to be able to assume that biogenesis could have been twice as long as $\Delta t_{\text {biogenesis }}$ (i.e., $N \geq 2$ ) to have interesting constraints: $q_{2}>0.13$, $q_{4}>0.36$ and $q_{10}>0.85$ at the $95 \%$ confidence level. The ability to extract a useful constraint even if $N$ is low reduces the influence of the non-observability-of-recent-biogenesis selection effect. However, estimates of how large $N$ is are problematic. If $N=1$ we can say nothing meaningful about $q_{1}(=q)$. See LD for details.

We convert this constraint into a constraint on the probability of biogenesis $q$ using a lottery model and some simple standard statistics (see LD for details).

For this conversion we need to include the fact that the evolution of an observer takes some time. How long it takes observing equipment to evolve, is difficult to say. On Earth it took $\sim 4$ Gyr. Thus, there is a selection effect 
favouring biogenesis to happen a few billion years before the present regardless of whether it happened rapidly. It is not a selection effect for rapid biogenesis since the longer it took us to evolve to a point when we could measure the age of the Earth, the older the Earth could become. For example, if biogenesis took 1 Gyr longer than it actually did, we would currently find the age of the Earth to be 5.566 Gyr $(=4.566+1)$ old.

Any effect that makes rapid biogenesis a prerequisite for life would undermine our inferences for $q$. For example, although it is usually assumed that the heavy bombardment inhibited biogenesis, energetic impacts may have set up large chemical and thermal disequilibria that play some crucial role in biogenesis. If true, the timescale of biogenesis would be linked to the timescale of the exponential decay of bombardment and biogenesis would (if it occurred at all) be necessarily rapid; most extant life in the Universe would have rapid biogenesis and little could be inferred about the absolute value of $q$ from our sample of one.

Carter (1983) has pointed out that the timescale for the evolution of intelligence on the Earth ( $\sim 4 \mathrm{Gyr})$ is comparable to the main sequence lifetime of the Sun $(\sim 10 \mathrm{Gyr})$. Under the assumption that these two timescales are independent, he argues that this would be unlikely to be observed unless the average timescale for the evolution of intelligence on a terrestrial planet is much longer than the main sequence lifetime of the host star (see Livio 1999) for an objection to the idea that these two timescales are independent). Carter's argument is strengthened by recent models of the terrestrial biosphere that indicate that the gradual increase of solar luminosity will make Earth uninhabitable in 1 or 2 billion years - several billion years before the Sun leaves the main sequence (Caldeira \& Kasting 1992).

Our analysis is similar in style to Carter's, however we are concerned with the appearance of the earliest life forms, not the appearance of intelligent life. Subject to the caveats raised by LD and Livio (1999), the implications of our analysis and Carter's are consistent and complementary: the appearance of life on terrestrial planets may be common but the appearance of intelligent life may be rare. Hanson (2002) applies Carter's reasoning about the emergence of intelligence (which took about 4 billion years) to the emergence of life (which may have taken 100 million years or less) and warns that the Earth may not be a random member of the planets with life - life had to evolve quickly to leave lots of time for intelligence to evolve. However, in the regime where $\Delta t_{\text {biogenesis }}$ is much less than the 1-2 billion years remaining for life on Earth (Caldeira \& Kasting 1992) this argument becomes less compelling.

\section{References}

Caldeira, K., \& Kasting, J. F. 1992, Nature, 360, 721

Carter, B. 1983, in Proc. R. Soc. Discussion Meeting on the Constants of Physics, ed. W. H. McRea \& M. J. Rees, R. Soc., and in Philos. Trans. R. Soc. London, A310, 347

Hanson, B. 2002, http://hanson.gmu.edu/vita.html

Lineweaver, C. H., \& Davis, T. M. 2002, Astrobiology, 2, 239

Livio, M. 1999, ApJ, 511, 429 